\title{
A Problem Involving Stresses In Solid Polymers
}

\author{
Patrick J. Cronin \\ The Pennsylvania State University \\ New Kensington Campus
}

\section{Introduction.}

Engineering materials books typically give a broad survey of different engineering materials such as ferrous metals, nonferrous metals, glass, plastics ( solid polymers ), etc. They give the advantages and disadvantages of each material. They give the mechanical properties of each material such as elastic modulus, tensile strength, Poisson's ratio, yield strength, etc.

The structure of solid polymers and their inherent displacement versus time properties are not usually included in such books. However, students in the mechanical design/analysis disciplines can benefit from an understanding of the stress and deformation of a solid polymer when external loads are applied to it. Many common engineering materials exhibit viscoelastic stress-strain properties. Some of these materials are: foam ear plugs, asphalt roads, wood structures, spinal disks, soil foundations, and gaskets.

\section{Structure of solid polymers.}

Solid polymers consist of a large number of very long strings of organic molecules such as shown in Figure $1^{1}$. The arrangement of these millions of long molecule chains is chaotic and intertwined. A schematic of such an arrangement is shown in Figure $2{ }^{2}$. An analogy would be a large clump of intertwined pieces of string or chain with lengths varying from moderately long to extremely long.

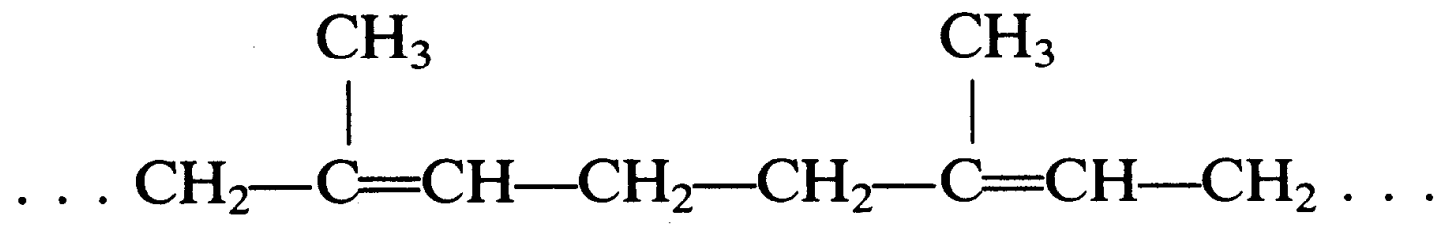

Figure 1. A typical solid polymer molecule. (From Reference 1, Dover Publications Inc., Reproduced with permission.)

When an external load is applied, there is often an immediate deformation due to the force applied to the intertwined molecules. Then there is a time dependent deformation due to the

"Proceedings of the 2003 American Society for Engineering Education Annual Conference \& Exposition Copyright (C) 2003, American Society for Engineering Education" 
complicated macroscopic structure of a solid polymer. This response can be considered to be a damped response because of the time delay of it. This phenomenon is used to apply solid polymers as vibration damping base supports for vibrating machinery. The solid polymer pads dampen the vibration energy due to the dampening property of their structure. For statically applied loads the deformation continues until the final deformation pattern is achieved. The time lag between the initial load application and the final deformation pattern can be less than a second.

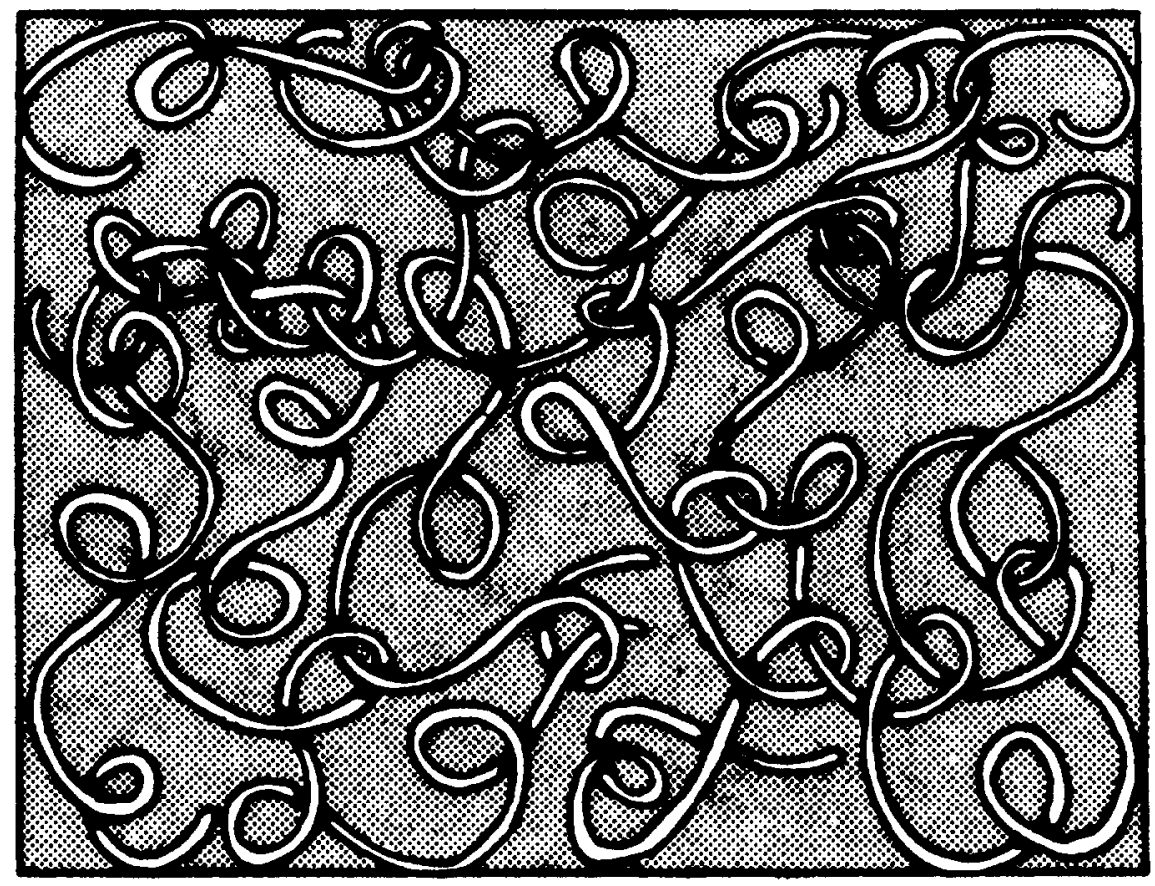

Figure 2. A schematic of solid polymer chains arrangement. (From Reference 2, John Wiley \& Sons, Ltd., Reproduced with permission.)

3. Mechanical models of solid polymers.

The deformation vs. load ( or strain vs. stress ) behavior of solid polymers mimics the deformation vs. load behavior of combinations of springs and dashpots. A few of the simpler arrangements are shown in Figure $3^{3}$. The first two are classified as two element models. The first is the Kelvin ( or Voigt ) model and the second is the Maxwell model. The third is a four-element model called Burgers model. These models can be used to represent the displacement vs. load behavior of some solid polymers over at least part of the load range. 
4. A solid polymer stress distribution example

The $\sigma_{\mathrm{r}}$ stress due to a concentrated load on a viscoelastic ( solid polymer ) half-space is ( in cylindrical coordinates ${ }^{4}$ :

$\sigma_{\mathrm{r}}(\mathrm{r}, \mathrm{z}, \mathrm{t})=$

$\frac{P_{\circ}}{2 \pi}\left\{\frac{3}{2 C+B}[B+2 C \exp [-(2 C+B) t / A]]\left[\frac{1}{r^{2}}-\frac{z}{r^{3}}\left(r^{2}+z^{2}\right)^{-\frac{1}{2}}\right]-3 r^{2} z\left(r^{2}+z^{2}\right)^{-\frac{5}{2}}\right\}, t>0$
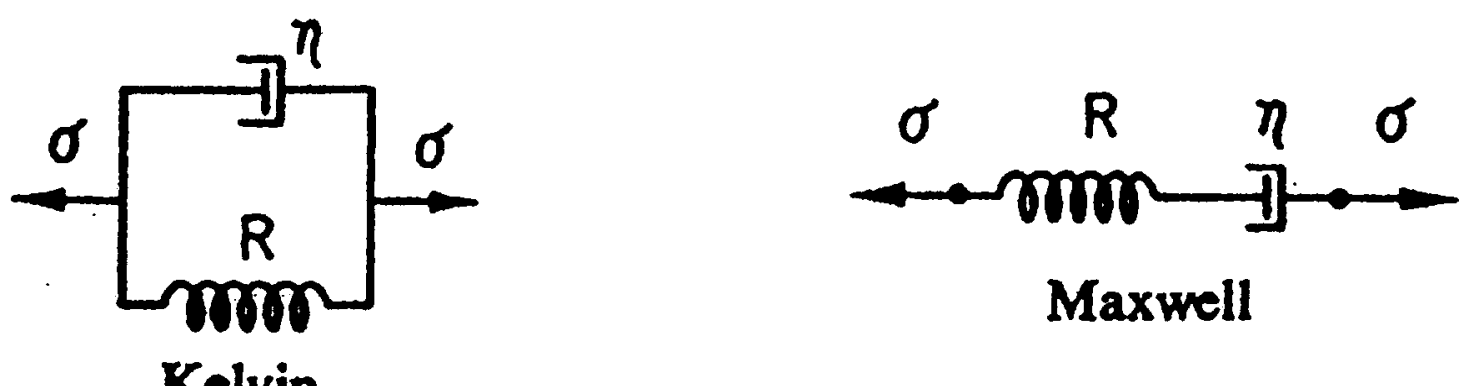

Kelvin

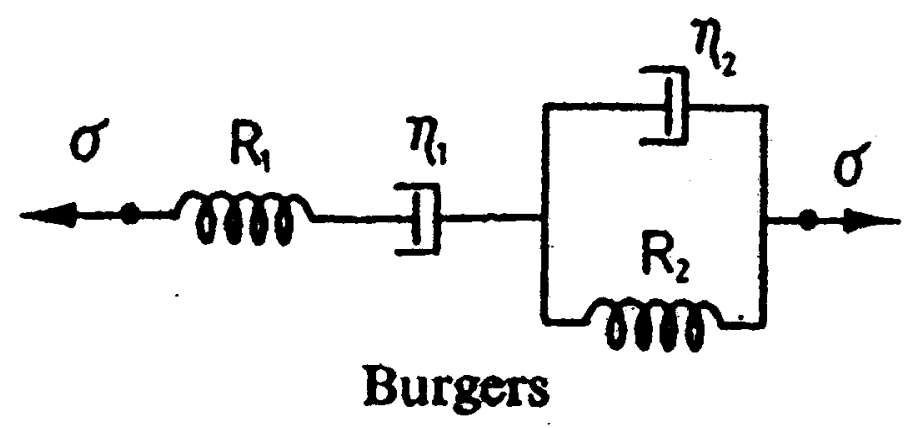

Figure 3. Some mechanical models of solid polymer deformation behavior.

(From Reference 3, Dover Publications Inc., Reproduced with permission.)

If the solid polymer behaves elastically based on average normal stress but as a Kelvin model (see Figure 3) based on shear stresses, then 
$\mathrm{A}=\eta($ damping constant $)$

$\mathrm{B}=\mathrm{R}$ ( shear elastic modulus )

$\mathrm{C}=\mathrm{K}$ (bulk modulus )

A problem was solved using some values for the material constants. Values for polymethyl methacrylate ( PMMA ) can be taken as ${ }^{5}$

$\eta=0.126 \frac{l b \cdot \sec }{i n^{2}}$

$\mathrm{R}=348 \quad \frac{l b}{i n^{2}}$

For calculational purposes, a value of $7.6 \times 10^{5} \mathrm{lb} / \mathrm{in}^{2}$ was used for K. A concentrated force of $P_{\mathrm{o}}=1.0$ pound was applied to the surface of the material. Table 1 shows the values of the $\sigma_{\mathrm{r}}$ stress during the deformation of the solid polymer.

Table 1. $\sigma_{\mathrm{r}}$ stress versus time during solid polymer deformation

\begin{tabular}{|c|c|c|}
\hline time $(\mathrm{sec})$ & $\begin{array}{c}\text { solid polymer } \\
\sigma_{\mathrm{r}}\left(\mathrm{lb} / \mathrm{in}^{2}\right)\end{array}$ & $\begin{array}{c}\text { elastic } \\
\sigma_{\mathrm{r}}\left(\mathrm{lb} / \mathrm{in}^{2}\right)\end{array}$ \\
\hline 0 & -4.28 & -4.66 \\
\hline $2 \times 10^{-5}$ & -4.38 & -4.66 \\
\hline $4 \times 10^{-5}$ & -4.45 & -4.66 \\
\hline $6 \times 10^{-5}$ & -4.50 & -4.66 \\
\hline $8 \times 10^{-5}$ & -4.54 & -4.66 \\
\hline $10 \times 10^{-5}$ & -4.57 & -4.66 \\
\hline $1.2 \times 10^{-4}$ & -4.60 & -4.66 \\
\hline $1.4 \times 10^{-4}$ & -4.61 & -4.66 \\
\hline $1.6 \times 10^{-4}$ & -4.62 & -4.66 \\
\hline $1.8 \times 10^{-4}$ & -4.63 & -4.66 \\
\hline $2 \times 10^{-4}$ & -4.64 & -4.66 \\
\hline
\end{tabular}

The stresses in the comparable elastic solid are shown as well. These values of the $\sigma_{\mathrm{r}}$ stresses are at $(\mathrm{r}, \mathrm{z})$ coordinate location $(0.1 \mathrm{in}, 0.01 \mathrm{in})$. The $\sigma_{\mathrm{r}}$ value at coordinate location $(0,0)$ is

"Proceedings of the 2003 American Society for Engineering Education Annual Conference \& Exposition Copyright (C) 2003, American Society for Engineering Education" 
infinite, but becomes finite at values of $\mathrm{r}$ and/or $\mathrm{z}$ greater than exactly 0.0 . A concentrated force is an idealization, but it can be used to approximate the near field and far field stresses due to a sharp tip of small radius on a very thick flat surface. It can be seen that the compressive stress in the solid polymer is initially less but that it grows exponentially until it finally reaches the same value as in the elastic solid. This represents the effect of the dashpot mechanical element. The dashpot element arises from the time delayed unwrapping of the solid polymer chains.

5. Extensions of the simple mechanical models.

Most solid polymers cannot be represented over the entire range of their load-displacement behavior by a mechanical model consisting of only a few springs and dashpots. A more complicated mechanical model consisting of many Maxwell units is shown in Figure $4{ }^{2}$.

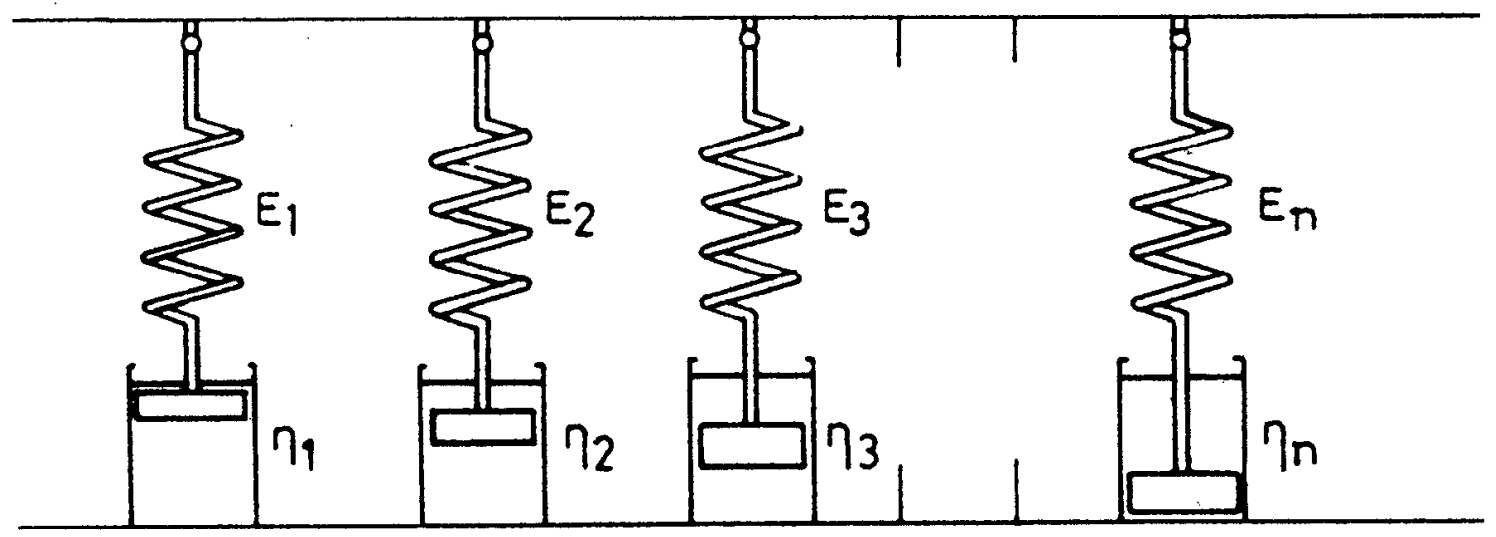

Figure 4. Mechanical model made up of many Maxwell units. (From Reference 2, John Wiley \& Sons, Ltd., Reproduced with permission.)

It can represent the load-displacement behavior of the solid polymer over a large range of time. Figure 5 ( upper curve ) shows ${ }^{6}$ the stress/strain ( $\log$ scale ) versus $\log t . E_{\mathrm{r}}(\mathrm{t})$ represents the stress relaxation modulus which is defined as $\sigma(\mathrm{t}) / \varepsilon$ where $\varepsilon$ is the applied constant strain. Figure 5 ( lower curve ) shows the distribution of relaxation times based on the $E$ and $\eta$ values of the various springs and dashpots needed to represent the response of the PMMA polymer. This $\mathrm{H}_{\varepsilon}(\tau)$ curve represents the relative influence of each particular Maxwell model on the $\mathrm{E}_{\mathrm{r}}(\tau)$ function. For instance, the peak value of $\mathrm{H}_{\varepsilon}(\tau)$ occurs at approximately $1 \times 10^{4}$ hours. Therefore, the Maxwell models with relaxation times around $1 \times 10^{4}$ hours have more influence on $\mathrm{E}_{\mathrm{r}}(\mathrm{t})$ than those with relaxation times around $1 \times 10^{-4}$ hours or $1 \times 10^{8}$ hours. 


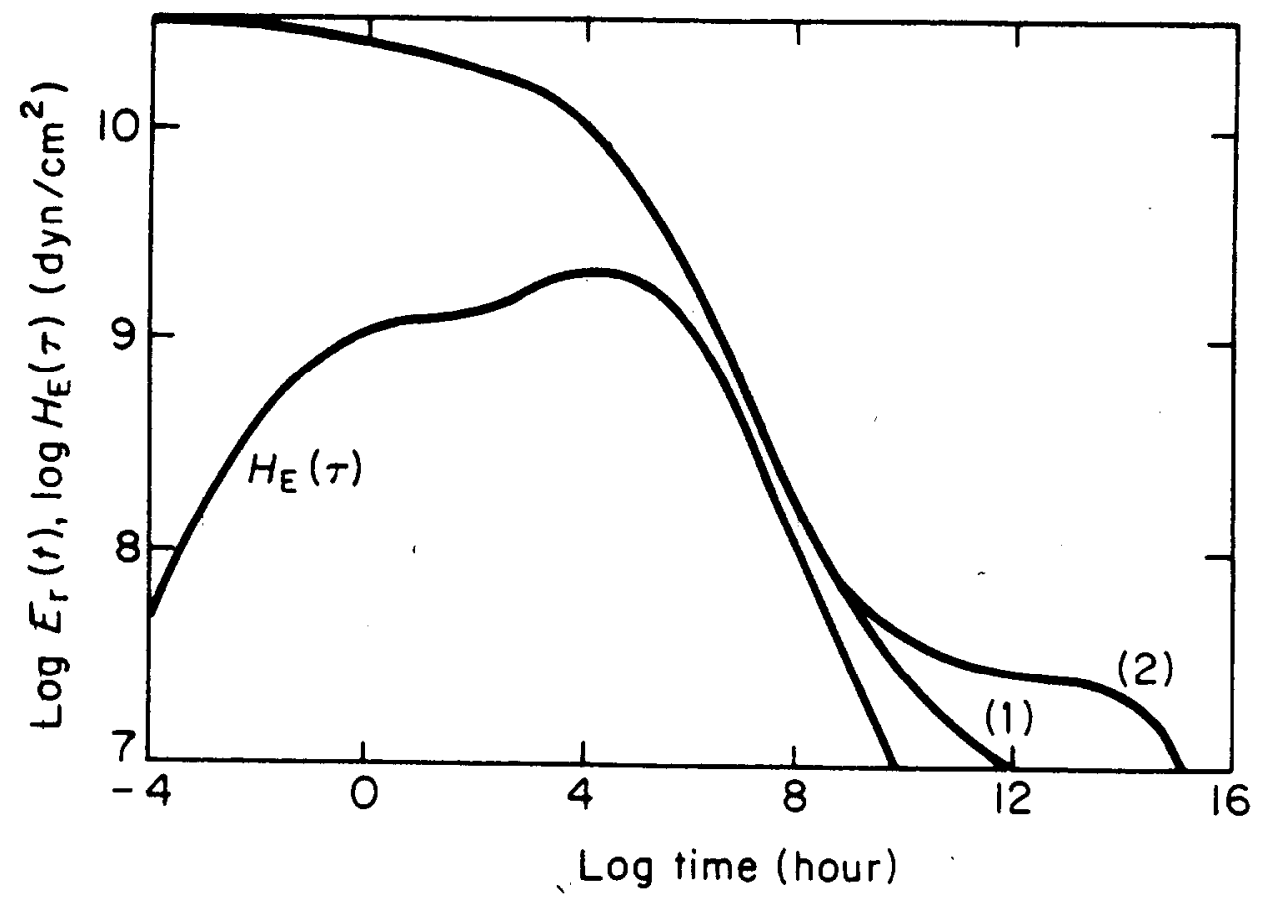

Figure 5. Stress/strain vs. $t$ and relaxation time spectrum for PMMA. (From Reference 6, Dover Publications Inc., Reproduced with permission.)

Values can be scaled from these curves for calculational purposes.

6. Summary.

Some qualitative aspects of the structure of modern plastics ( solid polymers ) have been presented here. They are suggested as possible items to be included in a course involving the choice of materials in mechanical design. There are numerous books and papers available which cover such topics in much more detail.

During the Spring 2003 semester I spent one to two class periods of my strength of materials course presenting to my class the information which I have outlined in this paper. They indicated to me that they felt that the material was interesting and worth the class time used.

Some aspects of the mechanical analogs of solid polymer deformation behavior are discussed. They are of some use when contemplating the deformation behavior. A solid polymer stress component which varies with time is presented to illustrate some quantitive behavior of a particular solid polymer when subjected to a concentrated force on its surface.

Strength of materials contains many formulas which are applied to determine the stress or stresses in various specific structural element cases. The equation which I am using in this paper is similar

"Proceedings of the 2003 American Society for Engineering Education Annual Conference \& Exposition Copyright (C) 2003, American Society for Engineering Education” 
to a strength of materials equation. Some differences are that it is a bit long and that it involves time as a variable. Some of the practical applications of this topic are an understanding of material behavior which leads to creep and stress relaxation in metals ( usually at elevated temperatures ) and some nonmetals of engineering importance.

References

1. Morawetz, Herbert, Polymers: The Origins and Growth of a Science, Dover Publications, Inc., New York, 1985 .

2. Ward, I.M. and Hadley, D.W., An Introduction to the Mechanical Properties of Solid Polymers, John Wiley and

Sons Ltd., U.K., 1993 .

3. Finley, W.N., Lai, J.S., and Onaran, K., Creep and Relaxation of Nonlinear Viscoelastic Materials, Dover Publications Inc., New York, 1989

4. Lee, E.H., Stress Analysis in Viscoelastic Bodies, Quarterly of Applied Mathematics, Volume 13, Number 2 ( p. 183 ), 1955.

5. Bland, D.R., The Theory of Linear Viscoelasticity, Pergamon Press, New York, 1960.

6. McCrum, N.G., Read, B.E., and Williams, G., Anelastic and Dielectric Effects in Polymeric Solids, Dover Publications Inc., New York, 1991.

\section{PATRICK J. CRONIN}

Patrick J. Cronin is currently an Assistant Professor of Engineering at The Pennsylvania State University New Kensington Campus. He holds a B.S. in Engineering Science and an M.S. degree in Civil Engineering both from the State University of New York at Buffalo. He previously worked as an engineer performing stress and vibration analysis of various types of mechanical equipment. 